but we observed slightly higher (by about 15-20\%) levels of myostatin and irisin in vegetarians.

Our results show that a well-planned vegetarian diet with proper dairy and egg intake does not lead to changes in bone mass in prepubertal children.

However, children following a vegetarian diet had a higher rate of bone turnover and subtle changes in serum myokine levels.

\section{DETERMINANTS OF ENERGY UNDER-REPORTING IN RURAL ADOLESCENTS}

Lyubov Rychkova*, Olga Dolgikh, Anna Pogodina, Zhanna Ajurova, Tatyana Astakhova. Scientific Centre for Family Health and Human Reproduction Problems

\subsection{6/archdischild-2021-europaediatrics.232}

To assess the extent of under-reporting (UR) in rural adolescents and investigate associated covariates.

A total of 150 adolescents aged 11-17 years were included. Food intake was reported in a $3 \mathrm{~d}$ diet record. Socio-economic status, sedentary behaviour and physical activity were collected by questionnaires. Weight height and waist circumference were measured. A body mass index (BMI) was calculated.

Plausibility cut-offs for reported energy intake as a percentage of predicted energy requirements were used to identify under-reporters.

Multivariate logistic regressions investigated the associations between UR and covariates.

The percentages of under- and over-reporters of energy intake were $35.3 \%$ and $3.3 \%$ respectively. Energy intake was under-reported more in older adolescents and boys. In multivariate analysis UR was associated with BMI and waist circumference Z-scores (OR 1,7 [95\% CI 1,1-2,4] and OR 1,5 [95\% CI 1,1-2,2] respectively) and participation in sports groups (OR 2,5 [95\% CI 1,1-5,4]).

In conclusion, in rural sample under-reporters differ from plausible reporters in several characteristics related to age, sex, weight status and organized physical activity. Therefore, it is important to consider this differential UR bias when investigating diet-disease associations in adolescents.

\section{TRANSITORY APLASTIC ANEMIA FOLLOWING ACUTE AUTOIMMUNE HEPATITIS IN A YOUNG BOY}

Matija Bakoš*, Vanja Ille, Matea Kovačić, Nedo Marčinković, Ana Petrović Gluščić, Jadranka Kelečić, Goran Tešović, Mirna Natalija Aničić, Lana Omerza, Irena Senečić Čala, Duška Tješić Drinković, Jurica Vuković. University Hospital Center Zagreb

\subsection{6/archdischild-2021-europaediatrics.233}

Introduction Acute hepatitis associated aplastic anemia (AHAAA) is a rare condition in which acute hepatitis is complicated by development of aplastic anemia. It is more common in young males, and presents with pancytopenia 2-3 months after an episode of acute hepatitis.

The etiology of hepatitis mostly remains unknown and it is thought that aplastic anemia is caused by immune dysregulation provoked by perhaps infection triggered cytokine production that injures hematopoetic stem cells. Options for treating severe AHAAA are bone marrow transplantation or immunosuppressive therapy (corticosteroids, cyclosporin A, antithymocyte globulin, antilymphocite globulin).

Case Report We present a case of a previously healthy 10-year old boy, admitted due to sudden onset of jaundice and nausea. Laboratory results showed highly elevated aminotransferase (AST $2089 \mathrm{U} / \mathrm{L}, \quad$ ALT $3228 \mathrm{U} / \mathrm{L}$ ), conjugated hyperbilirubinemia (total bilirubin 391 umol/L, conjugated 302 umol/L) and initially preserved synthetic liver function. Extensive workup excluded infectious, toxic and metabolic causes. Hypergammaglobulinemia was not present, but anti smooth muscle antibodies were positive in two consecutive testing (1:20). Liver biopsy showed unspecific mixed type acute inflammation. In the next few days he developed liver failure (INR 2.6) so immunosuppressive therapy for autoimmune hepatitis (AIH) was initiated (corticosteroids and azathioprine), together with symptomatic therapy (fresh frozen plasma).Due to potential adverse toxic effect (decreased activity of the enzyme thiopurinmethyltransferase) azathioprin was changed to mycophenolate mofetil. In the following weeks patient's synthetic liver function completely recovered and aminotransferase and bilirubin levels improved. Six weeks after initial onset of his symptoms patient developed severe thrombocytopenia and leukopenia followed by mild anemia. Bone biopsy showed hypoplastic to aplastic bone marrow. Screening for opportunistic pathogens revealed positive Pneumocystis carinii, and also CMV and VZV reactivation so treatment with trimethoprimsulfametoxazole and valganciclovir was started together with antifungal therapy for oral candidiasis. $\mathrm{He}$ also required repeated platelet transfusions. After two months his bone marrow started showing signs of recovery and he was discharged from hospital. At last follow up, his leukocyte counts was 4.3 x 109/L, hemoglobin $142 \mathrm{~g} / \mathrm{L}$ and platelet count 57 x 109/L. Discussion Our patient followed the typical presentation of AHAAA described in the scarce literature. The notable aspect of our case is potentially spontaneous recovery of bone marrow aplasia without the need for bone marrow transplantation. Mycophenolate mofetil might be viable therapeutic step in treatment for this type of disease.

\section{CASE OF FOREIGN BODIES IN GASTROINTESTINAL (GI) TRACT}

Paweł Józefczuk*, Izabella Łazowska-Przeorek, Katarzyna Karolewska-Bochenek Department of Paediatric Gastroenterology and Nutrition, Medical University of Warsaw Poland

\subsection{6/archdischild-2021-europaediatrics.234}

Rationale Foreign Body in GI Tract is an emergency state commonly treated in paediatric gastroenterology as well as paediatric surgery departments. Removal techniques (endoscopy vs. surgery) should be always carefully considered.

Patient An 8 year old boy was admitted to our Department because of intentional swallowing 9 magnetic balls (before 5 days). Abdominal pain, numerous vomiting and lack of appetite had been presented.

Intervention After X-Ray he was qualified to gastroduodenoscopy. 9 linearly connected magnetic balls were found. One boarder coated by pyloric mucosa and the second superficially fused with anterior wall of duodenal bulb.

Foreign bodies were successfully removed with Roth net. We discovered horizontal transmucosal ulcer in pylorus and deep penetrating ulcer of duodenal bulb with suspicion of its microperforation. 